\title{
A NUMERICAL INVESTIGATION INTO THE BEHAVIOR OF FLEXIBLE UPLIFTING STRUCTURES WITH SLIP-FRICTION CONNECTORS UNDER EARTHQUAKE LOADING
}

\author{
Wei Y. Loo, Pierre Quenneville, and Nawawi Chouw \\ Department of Civil and Environmental Engineering, University of Auckland \\ Symonds Street, Auckland, New Zealand \\ e-mail:wloo002@aucklanduni.ac.nz
}

Keywords: Slip-friction connector, uplift, rocking structure, earthquake engineering.

\begin{abstract}
While there has been significant research into the behavior of uplifting (or rocking) rigid structures under seismic loading, far less attention has been paid to the behavior of flexible structures allowed to uplift. Recently, however, Acikgoz and DeJong [1] have shown that these types of structures have unique dynamic characteristics that arise from the interaction of rocking motion with elastic vibration. An understanding of the behavior of uplifting flexible structures is required in order to fully benefit from the damage avoidance potential of allowing uplift. This is because building structures are invariably flexible with a finite fundamental frequency, and it is the desire of the structural designer that the structures themselves remain elastic, even while displaying non-linear behavior in a global sense. This paper is concerned with the behavior of flexible uplifting structures adapted with slip-friction devices. These devices act as hold-down connectors resisting overturning moment. They have a definite force threshold at which sliding will occur, and exhibit close to perfect elasto-plastic behavior. The ability of shear walls to avoid damage through the use of slip-friction connectors has been previously demonstrated by Loo et al. [2]. The modeling of the slip-friction connectors, using a combination of elements commonly available in finite element software packages, and their verification is discussed. Both single-degree-of-freedom (SDOF) and multi-degree-of-freedom (MDOF) flexible structures were modeled with a single plastic deformable hinge at their bases, and their behavior under various earthquake simulations investigated. These flexible structures (both SDOF and MDOF) were then affixed at their bases to a rigid platform, with slip-friction connectors implemented at the ends of the platform, and then subjected to the same earthquake simulations. It was found that the maximum drifts of structures with slipfriction connectors compared favorably with their equivalent structures with a single plastic deformable hinge. With a small amount of vertical load, residual drifts were also found to be small. The performance of MDOF structures with slip-friction connectors was somewhat superior to that of the SDOF structures, with maximum drifts and residual drifts smaller than that of comparable SDOF structures.
\end{abstract}




\section{INTRODUCTION}

In recent years there has been increasing interest in designing structures in earthquake prone areas that have the ability to avoid damage, and to re-centre after a seismic event. Earthquake resistant structures should thus allow not only for life preservation, but also avoid sacrificial damage.

One way of limiting damage on a structure during an earthquake is to allow it to uplift or to rock. Historically most of the research on rocking structures has concentrated on the behavior of rigid rocking blocks. However Acikgoz and DeJong [1] have recently written on the unique behavior of rocking flexible structures. This has applicability to the future design of rocking structures, because building structures are invariably flexible and not rigid, with a finite fundamental frequency of vibration.

An additional embellishment on the concept of a rocking flexible structure is the use of slipfriction dampers to provide damping and to cap earthquake induced forces on a structure below desired level. Slip-friction connectors (also commonly known as slotted-bolt connectors) can be fabricated in such a way that their hysteretic behavior approaches that of the idealized square shaped loops characteristic of a perfectly elasto-plastic substance.

In this paper a brief overview of slip-friction connectors is provided. These connectors are numerically implemented in models of flexible rocking structures, and their effectiveness in protecting both SDOF and MDOF structures from high base shears, and their performance in terms of maximum and residual drifts is demonstrated.

\section{SLIP-FRICTION CONNECTORS: CONCEPT AND MODELING}

\subsection{Concept}

Slip-friction connectors have in recent years been used or tested in both moment resisting frames and steel braced frames. Butterworth [3] describes their use in concentrically X-braced frames, and found from a preliminary time history analysis, that they had the ability to significantly increase the ductility of these frames. Butterworth et al. [4] also describe a particular type of slip-friction connector called the sliding hinge joint. The sliding hinge joint is used at the beam to column joint in moment resisting steel frames. During earthquake loading, the sliding hinge joint simulates yielding and plastic hinging by allowing sliding between steel plates - but without the concomitant permanent damage normally expected with traditional moment resisting steel frames after a design level seismic event.

Loo et al. [2], has demonstrated the performance advantages of the use of slip-friction connectors as hold-downs in timber shear walls. Base shears are limited to a predictable level, damage to the sheathing to framing nail connections is largely avoided, and re-centring performance found to be acceptable.

Slip-friction connectors are simple in configuration. The connector of Figure 1 has been proposed by the authors and consists of abrasion resistant steel plate sandwiched between outside plates of mild steel. The mechanism of sliding is symmetrical, in that the centre plate takes the connector force, $\mathrm{F}_{\text {slip }}$, and each outside plate exactly $\mathrm{F}_{\text {slip }} / 2$. 


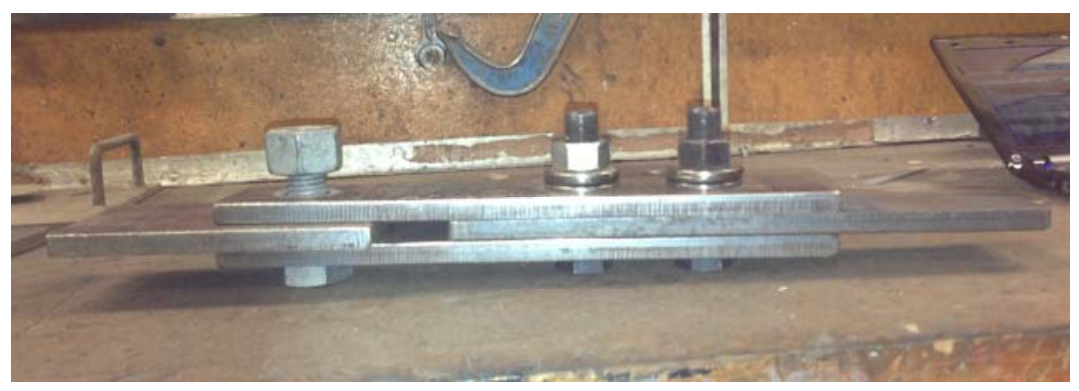

Figure 1: Slip-friction connector

The connector force, $\mathrm{F}_{\text {slip }}$, is a function of the number of bolts, $\mathrm{n}_{\mathrm{b}}$, clamping the plates of the connector together, the tension in each of the bolts, $\mathrm{T}_{\mathrm{b}}$, and the coefficient of friction, $\mu$.

$$
F_{\text {slip }}=2 n_{b} T_{b} \mu_{f r}
$$

In this paper slip-friction connectors are proposed for use as hold-downs for a rocking structure, and are implemented at the bottom corners of these structures.

\subsection{Modeling}

The behavior of a generic slip-friction connector can be readily modeled in most finite element software packages through using a multi-linear plastic nonlinear link, combined in parallel with a gap element. The multi-linear plastic element, by itself, would allow the model connector to displace upwards (positive displacement) and downwards (negative displacement) in an elasto-plastic manner.

However, for the bottom corners of a rocking structure, only uplift and its associated downward return motion is permitted - because the corner of the wall should not displace below its original level. Thus, the slip-friction connector must prevent displacement below the original position of the node to which it is attached. In order to do this a gap element is placed in parallel with the multi-linear plastic element. The initial 'gap' of the gap element is set to zero i.e. the gap is closed unless until there is a force causing uplift at its position. For positive displacement the stiffness is set to zero. For negative displacement a large value for stiffness is defined in order to keep any downward displacement to a negligible amount. To limit the amount of uplift, a hook element, is sometimes added to the connector assemblage. However for this study no limit was placed on uplift, thus allowing the maximum drifts that would naturally occur through rocking to be studied. Therefore the hook element was not required. The combined force displacement behaviors of the multi-linear plastic element and gap element produces hysteric behavior of the form shown in Figure 2 (taken from the SDOF slip-friction case of Section 3):

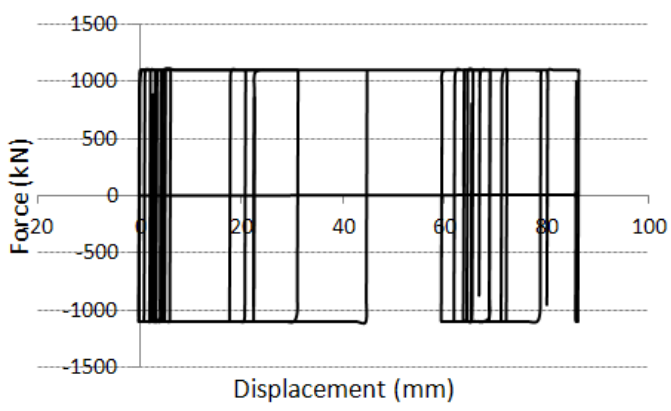

Figure 2: Force displacement behavior of numerically modeled slip-friction connector. 
For a more detailed description of the modeling of slip-friction connectors, the reader is referred to Loo et al. [2].

\section{EXPLORING THE BEHAVIOR OF STRUCTURES WITH SLIP-FRICTION CONNECTORS}

\subsection{Overview}

In this section a set of results from a wider study carried out on SDOF and MDOF structures with slip-friction connectors is presented. The earthquake records used are first discussed, the modeling procedure described, and results are presented.

\subsection{Acceleration records}

The ground excitations used in the simulations were based on the design spectra adopted in Japan following the destructive 1995 Kobe earthquake. These spectra (5\% damping) (see Figure 3) were used for this research because they have a distinct dominant frequency range, allowing relative ease in the interpretation of results. The return period for earthquake records used is 2500 years, which typically corresponds to a maximum considered event (MCE).

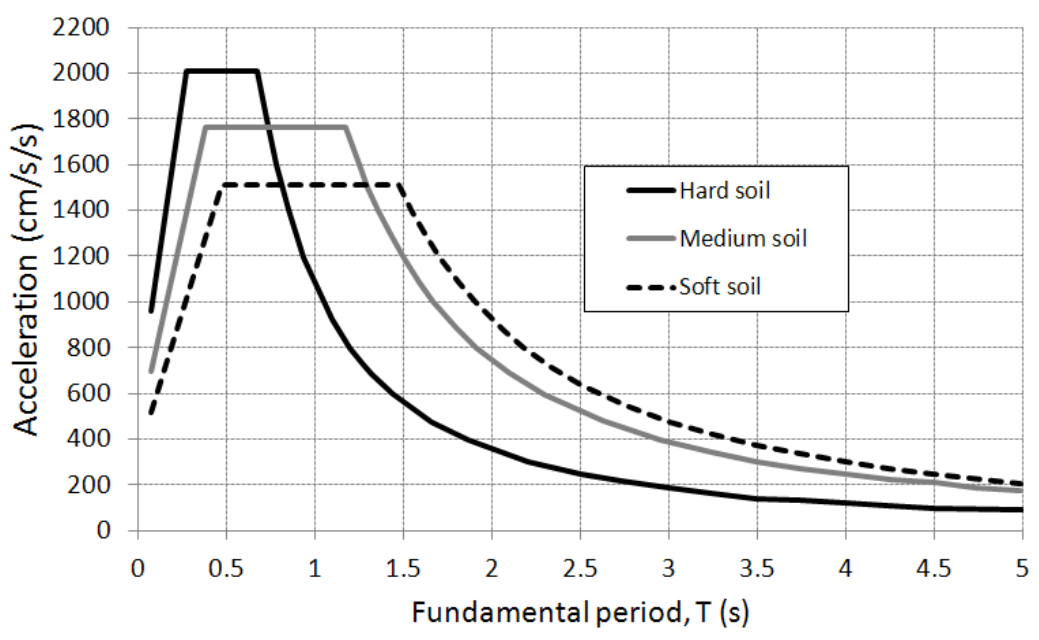

Figure 3: Japanese design spectra for soft, medium, and hard soil conditions.

Ninety ground acceleration records were used in this study; with thirty records for each of soft, medium, and hard soil conditions. In this paper, only the results for an SDOF and MDOF structure, both of fundamental period $0.55 \mathrm{~s}$, and subjected to ground accelerations associated with medium soil conditions is presented.

\subsection{Numerical modeling and results}

For both the SDOF and MDOF cases, simulations were carried out on flexible structures with (1) a rigid joint at the base, then (2) a plastic deformable joint at the base, then (3) slipfriction connectors at the base, and finally (4) slip-friction connectors at the base and vertical loading applied to the top of the structure. The SDOF structures are modeled as a $9.6 \mathrm{~m}$ high flexible column with a single mass at the top of the column for the SDOF structures, while the MDOF structures have a total height of $16 \mathrm{~m}$, with masses evenly distributed up the column (refer Figure 4). 


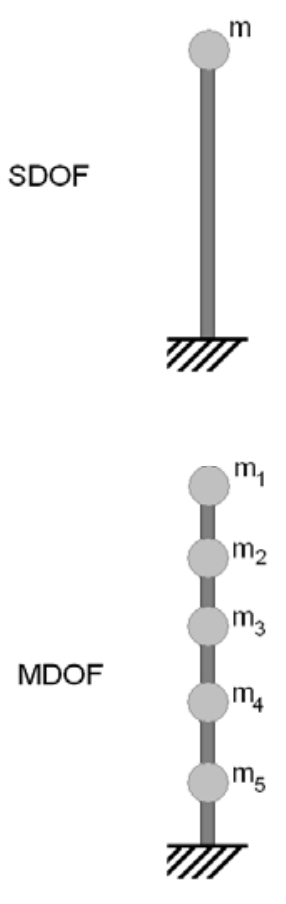

(a)
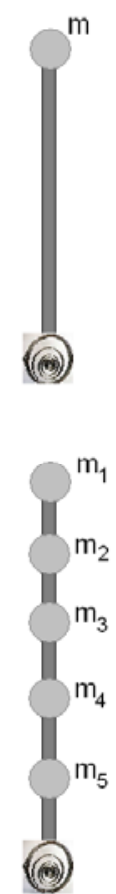

(b)
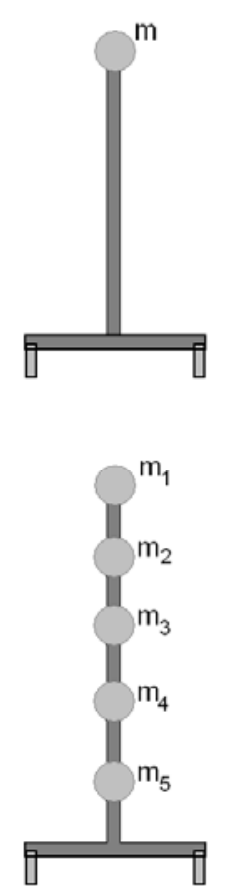

(c)

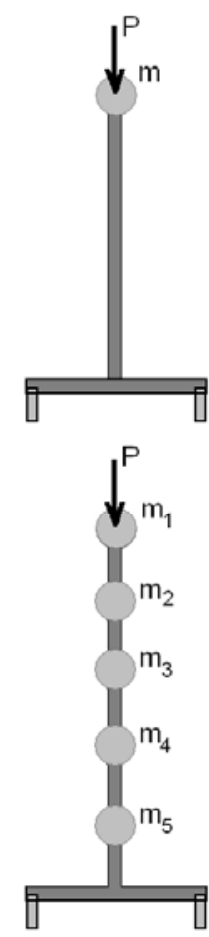

(d)

Figure 4: Modeling of SDOF structures (top row) and MDOF structures (bottom row): (a) fully elastic case, (b) ductile with plastic deformable hinge, (c) ductile with slip-friction connectors, and (d) ductile with slip-friction connectors and vertical load.

For the case considered, the Japanese design spectral response acceleration for a structure with fundamental period of $0.55 \mathrm{~s}$ is $17.6 \mathrm{~m} / \mathrm{s}^{2}$. For the SDOF case a mass of $125000 \mathrm{~kg}$ was used and the EI and length of the column adjusted to provide for the desired period of $0.55 \mathrm{~s}$. For the MDOF case, masses of $25000 \mathrm{~kg}$ were provided at each level. The EI of the column was adjusted to provide a fundamental period of $0.55 \mathrm{~s}$, with the length being $16 \mathrm{~m}$.

For the SDOF structure, the expected base shear for the fully elastic case, $\mathrm{V}_{\text {base }}$ was found simply by multiplying the response acceleration by the lumped mass atop the column. Thus $\mathrm{V}_{\text {base }}$ was found to be $2163 \mathrm{kN}$. Subjecting this structure with a fully rigid joint at the base to earthquake excitations produced an average base shear of $2160 \mathrm{kN}$, very close to the expected.

For the SDOF structure with the plastic deformable hinge, a force reduction factor of $\mathrm{R}=4$ was adopted. Therefore the target $\mathrm{V}_{\text {base }}$ was $541 \mathrm{kN}$. Given that the model height adopted was $9.6 \mathrm{~m}$, the maximum overturning moment $\mathrm{M}_{\mathrm{o}}$ was thus $541 \mathrm{kN}$ x $9.6 \mathrm{~m}=5194 \mathrm{kNm}$. A multilinear plastic link was used to model the behavior of this plastic deformable hinge, and its moment-rotation behavior defined. For the case with slip-friction connectors, the width of the rigid base of the structure was $4.8 \mathrm{~m}$. Hence to resist overturning moment, the slip force, $\mathrm{F}_{\text {slip, }}$, of the connector was set to be $\mathrm{M}_{\mathrm{o}} / 4.8 \mathrm{~m}=1082 \mathrm{kN}$. The moment-rotation and forcedisplacement relationships for the plastic deformable hinge and the slip-friction connectors are shown in Figure 5. 

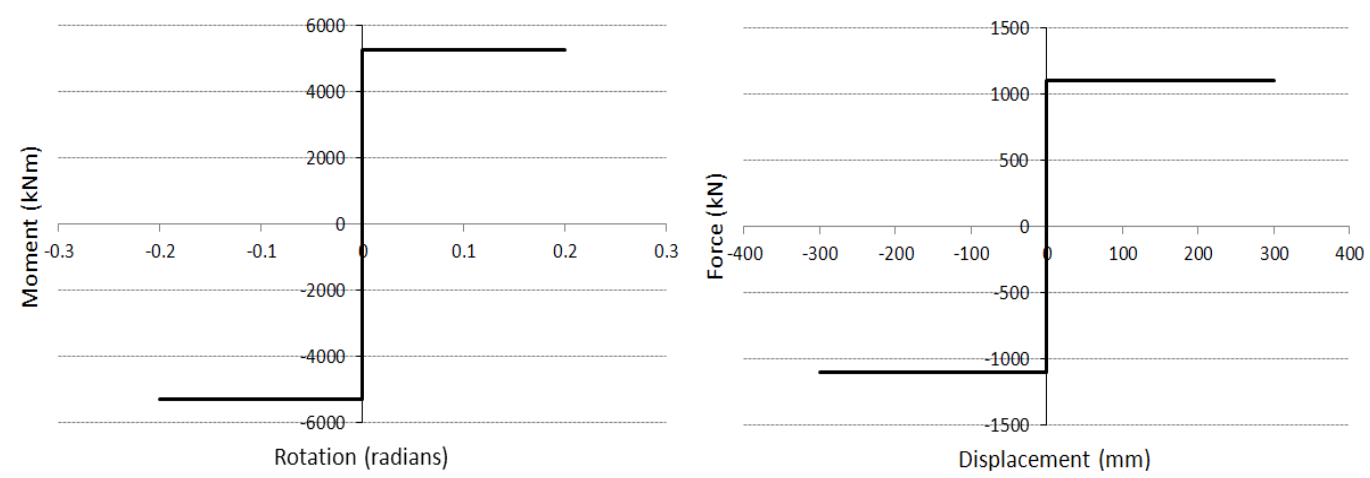

Figure 5: (a) Moment-rotation behavior of plastic deformable hinge, and (b) Force-displacement behavior of slip-friction connector.

For the case of the SDOF structure with vertical loading, it was decided to load the structure in such a way that the resistance to overturning moment provided by the vertical load would be $15 \%$ of the resistance to overturning moment provided by the slip-friction connectors. Thus a vertical load, $\mathrm{P}$, of $287 \mathrm{kN}$ was adopted, and the slip-friction connector force, $\mathrm{F}_{\text {slip }}$ set to $957 \mathrm{kN}$. Thus the moment resistance of the vertical load is $287 \mathrm{kN} \mathrm{x} 2.4 \mathrm{~m}=689 \mathrm{kNm}$, while that of the slip-friction connectors is $957 \mathrm{kN}$ x $4.8 \mathrm{~m}=4594 \mathrm{kNm}$.

Figure 6 shows the base shears for the SDOF structure, with the fully elastic case shown first, and then the load limiting effects of the plastic deformable hinge, the slip-friction connectors by themselves, and finally the combination of slip-friction connectors with vertical load. It can be seen that for the ductile cases, the base shear is capped to the desired level of around $540 \mathrm{kN}$.
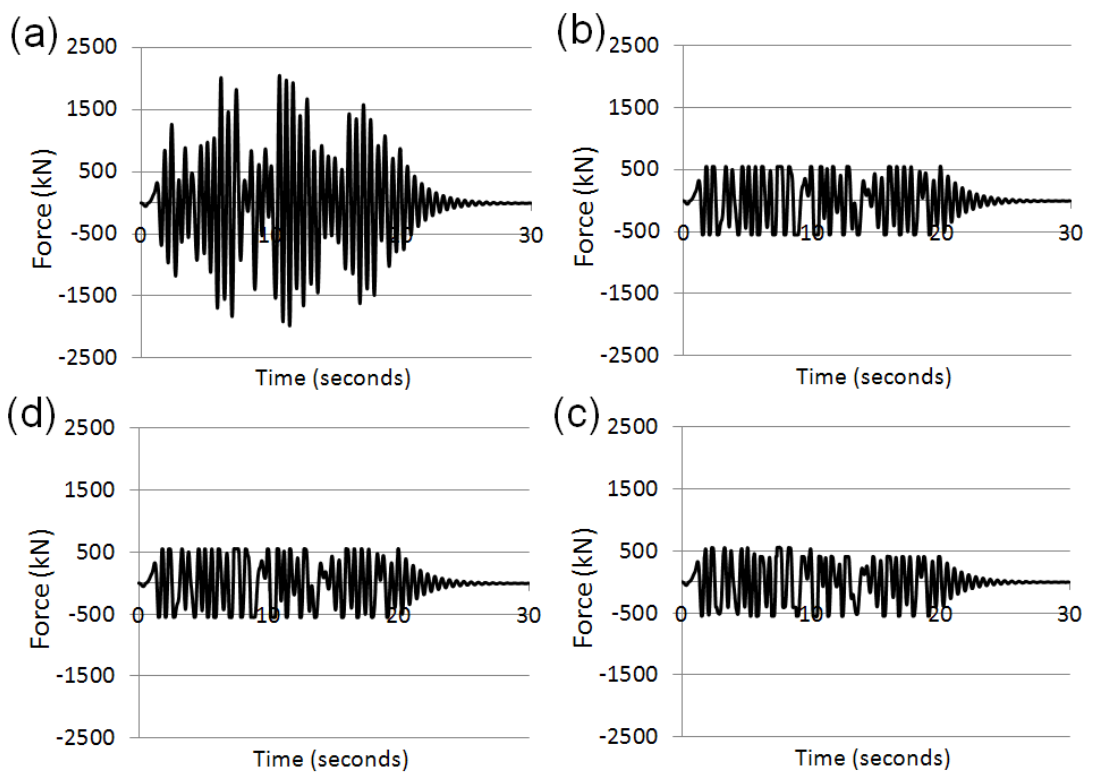

Figure 6: Base-shear time history of SDOF structures - (a) fully elastic case, (b) ductile with plastic deformable hinge, (c) ductile with slip-friction connectors, and (d) ductile with slip-friction connectors and vertical load.

When modeling the MDOF structures, a similar approach was taken to that of the SDOF case, except the column was lengthened from $9.6 \mathrm{~m}$ to $16 \mathrm{~m}$, and the $125000 \mathrm{~kg}$ mass distributed over 5 levels. The $1^{\text {st }}$ to $5^{\text {th }}$ modes of vibration are shown in Figure 7. 


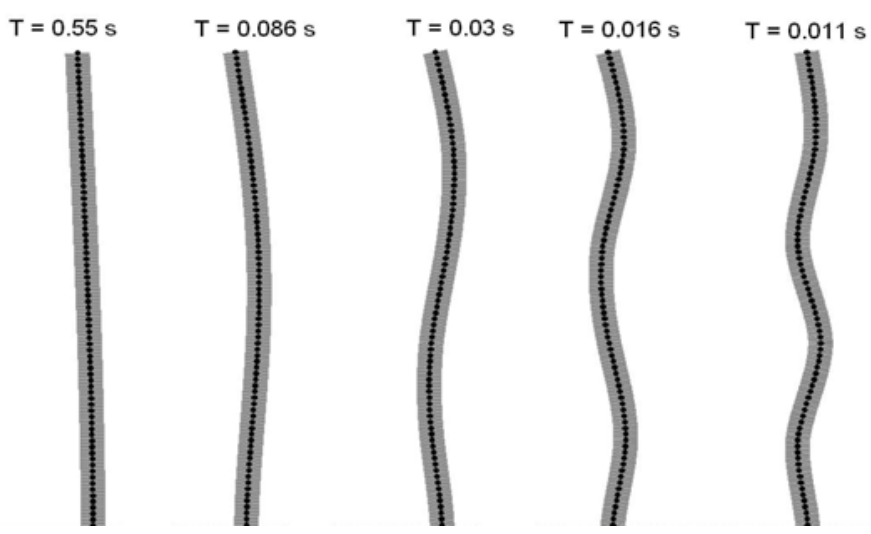

Figure 7: Modes of vibration for MDOF structure

Results for maximum horizontal and residual displacements at the top of the MDOF and SDOF structures, are now discussed. For the example presented in this paper, it can be seen that allowing ductile behavior, for both the MDOF and SDOF cases, results in displacements that are higher than that of the fully elastic cases (see Figure 8).

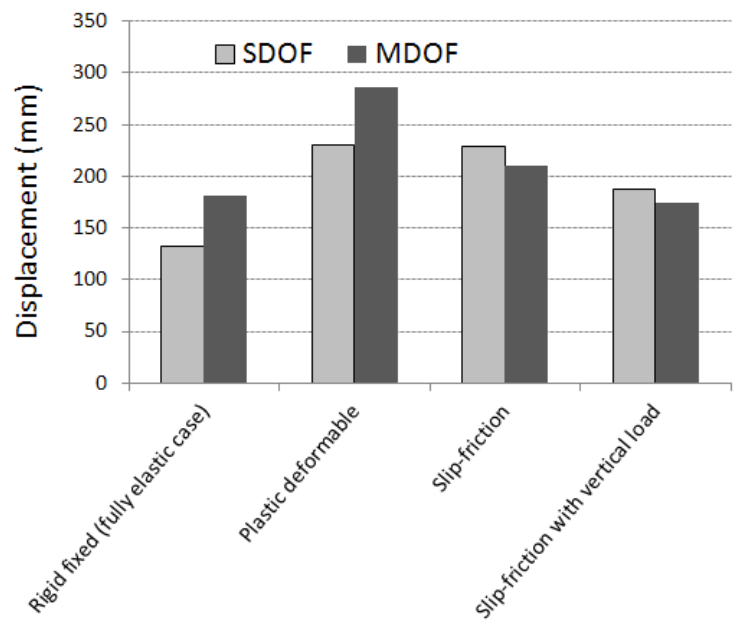

Figure 8: Maximum displacements at top of structure under earthquake loading.

This, however, is expected, as the fundamental period of $0.55 \mathrm{~s}$ for both types of structures is within the range where maximum inelastic displacements under earthquake loading are expected to exceed the elastic spectral displacements. From Figure 8 it can be seen that for both the MDOF and SDOF cases maximum drifts are less for structures in which base shears are capped by slip-friction connectors, compared with the case for those structures in which ductility is supplied by a plastic deformable hinge. Where slip-friction connectors are utilized, the drifts for the MDOF structure are slightly smaller than that of the equivalent SDOF structure.

For residual drifts, the application of vertical load to assist in resisting overturning moment appears to suppress residual drift to very low levels (see Figure 9). This is in spite of the fact that the moment resistance of the vertical loading was only $15 \%$ that of the moment resistance from the slip-friction connectors. 

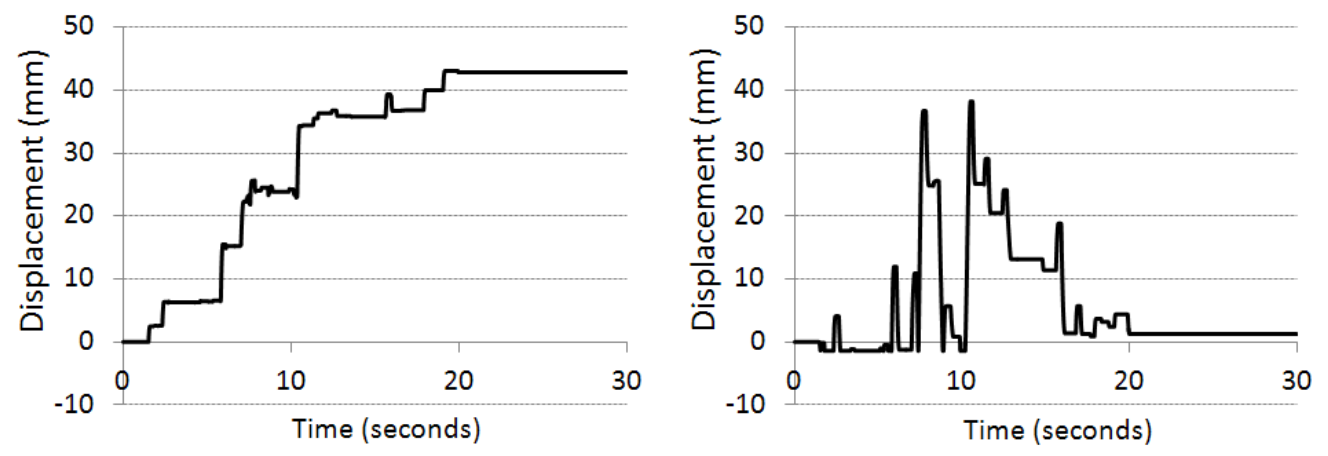

Figure 9: The effect of vertical loading on residual displacement (a) displacement time-history of structure without vertical loading and (b) with vertical load.

From Figure 10 it can be seen that for the case considered, slip-friction connectors provide for smaller residual drifts than the equivalent case with a plastic deformable hinge, and that the effect is more pronounced in the case of the MDOF structure.

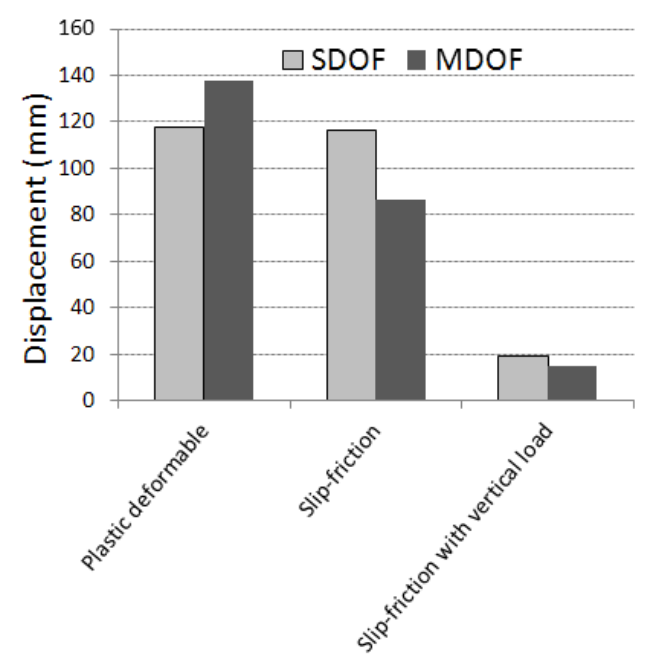

Figure 10: Residual drifts.

\section{CONCLUSIONS}

Some results from a wider study on the comparative performance of flexible SDOF and MDOF structures with slip-friction connectors are provided. For ductile structures, slipfriction connectors appear to have lower drifts in general, than that of the case where ductility is provided by a single plastic deformable hinge. Residual drifts are also smaller in the case of ductile structures with slip-friction connectors. The application of vertical loading has a relatively minor effect on reducing maximum displacement, but does significantly suppress residual displacements for both SDOF and MDOF structures. Residual drifts and maximum drifts for the MDOF structures appear to be less, under the same earthquake loadings, than those associated with comparable SDOF structures.

\section{ACKNOWLEDGMENTS}

The authors would like to thank the New Zealand Ministry for Primary Industries for the support of this research. 


\section{REFERENCES}

[1] S. Acikgoz, M. J. DeJong, The interaction of elasticity and rocking in flexible structures allowed to uplift. Earthquake Engineering and Structural Dynamics, 41(15), 2177-2194, 2012.

[2] W. Y. Loo, P. Quenneville, N. Chouw, A numerical study of the seismic behaviour of timber shear walls with slip-friction connectors. Engineering Structures, 34(22), 233243, 2012.

[3] J. Butterworth, Ductile concentrically braced frames using slotted bolted joints. Journal of the Structural Engineering Society of New Zealand, 13(1), 39-48, 2000.

[4] J. Butterworth, C. G. Clifton, G. MacRae, Developments in steel frame joints in New Zealand. The Structural Engineer, 86(16), 20-21, 2008. 